\title{
Theoretical studies of the applications of resonant tunneling diodes as intersubband laser and interband excitonic modulators
}

\author{
J. P. Loehr, J. Singh, R. K. Mains, and G. I. Haddad \\ Center for High Frequency Microelectronics, Department of Electrical Engineering and Computer \\ Science, Universily of Michigan, Ann Arbur, Michigun 48109-2122
}

(Received 18 March 1991; accepted for publication 29 July 1991)

\begin{abstract}
We present a theoretical analysis of the optical applications of resonant tunneling diodes. The electronic properties are calculated with a self-consistent traveling-wave model that includes effective-mass mismatches. The interband optical properties are calculated from a $4 \times 4 \mathrm{k} \cdot \mathrm{p}$ band structure in the dipole approximation. We find that it is possible to operate a conventional device as an intersubband laser if the transition energy is large $(\sim 0.5$ $\mathrm{eV})$ and the linewidth in minimal ( $\sim 5 \mathrm{meV})$. A bound-state device can produce a modulation ratio of 5:1 at the excitonic peak with an absorption length of $\sim 40 \mu \mathrm{m}$ in a waveguide geometry.
\end{abstract}

In this work we analyze the feasibility of lasers and modulators which use resonant tunneling diodes (RTDs) in the active region. The laser structure involves optical transitions between conduction subband states while the modulator involves excitonic transitions. We consider two devices for our study. The conventional structure [Fig. 1(a)] has $\mathrm{In}_{0.53} \mathrm{Ga}_{0.47}$ As contacts, $23 \AA$ AlAs barriers and a $55 \AA \mathrm{In}_{0.53} \mathrm{Ga}_{0.47} \AA$ s well; the entire structure is $n$ doped at $2 \times 10^{18} \mathrm{~cm}^{-3}$. We will be interested in intersubband transitions in this structure; hence, we have used very high AlAs barriers and an $\mathrm{In}_{0.53} \mathrm{Ga}_{0.47}$ As well to obtain a large enough band discontinuity to get two conduction subbands in the narrow well region. We consider operating this device as an intersubband laser. The bound-state structure [Fig. 1(b)] has GaAs contacts, $28 \AA \mathrm{Al}_{0.30} \mathrm{Ga}_{0.70} \mathrm{As}$ barriers and a $67 \AA \mathrm{Al}_{0.25} \mathrm{Ga}_{0.75} \mathrm{As}$ well; the contacts are $n$ doped at $2 \times 10^{18} \mathrm{~cm}^{-3}$; the well and the barriers are undoped. Since the well band gap is lower than that of the heavily doped contact regions, the well is filled with bound carriers at zero bias. We consider operating this device as an excitonic modulator. We calculate the electronic properties of these devices via a self-consistent traveling-wave model that includes effective mass mismatches between the well and barriers; the contact regions are treated using a Thomas-Fermi model. ${ }^{1-3}$

It has been suggested ${ }^{4-7}$ that the intersubband transitions between the two resonant levels in the conduction band of a conventional RTD could be used to produce an electromagnetic source in the infrared region. Since the transitions occur between parabolic subbands, all of the transitions are at the same energy and the photon stimulated emission rate (or material gain) is simply proportional to the difference (or population inversion) between the electron concentrations in the first and second subband states. When the device is biased at the second resonant state, there can be a large population inversion in the well region, as shown in Fig. 2. The material gain for this twolevel system is given in mks units by ${ }^{8}$

$$
g=\frac{\Delta N_{21}(\hbar \omega)\left|M_{21}\right|^{2} q^{2} \cos ^{2} \theta\left(\hbar / \tau_{21}\right)}{n_{r} c \epsilon_{0} \hbar d\left[\left(E_{2}-E_{1}-\hbar \omega\right)^{2}+\left(\hbar / \tau_{21}\right)^{2}\right]}
$$

Here $\Delta N_{21}$ is the difference in carrier concentrations between the first and second subbands, $\hbar \omega$ is the photon energy, $\theta$ is the angle of photon polarization relative to the growth direction $z, n_{r}$ is the refractive index, $\epsilon_{0}$ is the mks free-space permittivity, $c$ is the velocity of light in vacuum, $d$ is the quantum well width, $\left(E_{2}-E_{2}\right)$ is the energy difference between the subbands, and $\tau_{21}$ is the intersubband relaxation time. The matrix element is given by
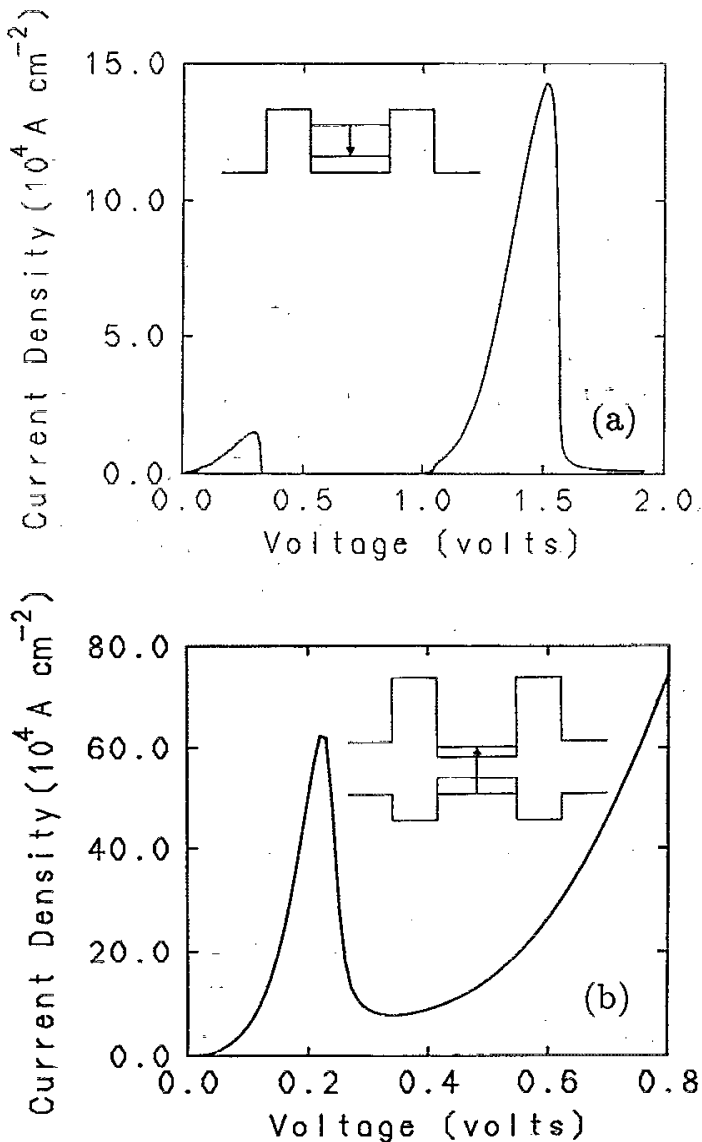

FIG. 1. Calculated current-voltage characteristics for the (a) conventional and (b) bound-state RTDs. 


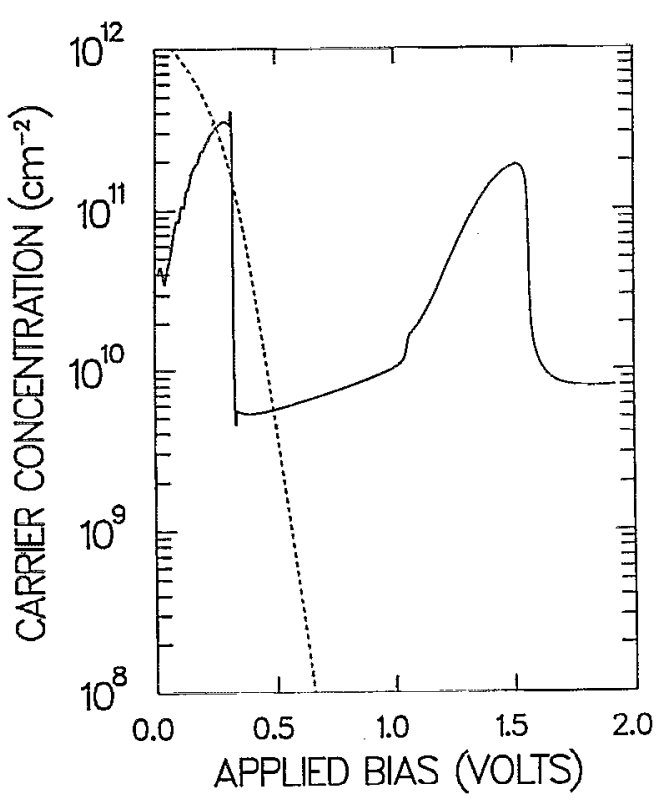

FIG. 2. Carrier concentrations in the wcll of the conventional (solid curve) and bound-state (dashed curve) devices as a function of applied bias. The dashed curve represents only the bound carrier concentration, the solid curve the total carrier concentration. In the first peak (resonance) of the solid curve essentially all of the carriers are in the first subband; in the second peak (resonance) of the solid curve essentially all of the carriers are in the second subband.

$$
M_{21}=\int d z \Phi_{2}^{*}(z) z \Phi_{1}(z),
$$

where $\Phi_{i}(z)$ is the $z$ dependence of the envelope furction of state $i$. Note that the gain is maximized for light polarized perpendicular to the layers ( $\widehat{z}$ polarization).

We obtain the carrier concentrations in the well from self-consistent calculations of the current-voltage characteristics of the structure, using a traveling-wave simulation and integrating the total electron concentration. Within this technique it is extremely difficult to extract, indeed even to define, the carrier concentrations in each separate subband. However, when the device is biased at a resonance, the energy distribution of the quasibound carriers is very sharply peaked at the energy of the resonant subband, and it is reasonable to assume that essentially all of the carriers are "in" the resonant subband. Hence, when the device is biased at the second resonance we assume that all of the carriers are in the second subband and we taken $\Delta N_{21}$ to be the total carrier concentration in the well $\left(\sim 1.9 \times 10^{11} \mathrm{~cm}^{-2}\right.$ from Fig. 2). In a similar approximation, the wave functions at the most probable energies in the first and second resonances are used to calculate $M_{21}$. We take $\tau_{21}$ to be $0.14 \mathrm{ps,} \mathrm{in} \mathrm{reasonable} \mathrm{agreement} \mathrm{with}$ experimental results. ${ }^{9}$ There is some uncertainty in this value, however, and the peak value of the gain is directly proportional to this parameter. Hence, extremely highquality material is necessary to reduce the linewidth as much as possible. For the structure and material parameters described above we obtain a $\hat{z}$-polarized $(\theta=0)$ peak gain of $15300 \mathrm{~cm}^{-1}$ at a photon energy of $0.533 \mathrm{eV}$.

In order for this system to operate as a separate con- finement heterostructure laser embedded in a Fabry-Perot cavity, it must satisfy the well-known lasing condition on the material gain $g$ :

$$
\Gamma_{g}=\frac{1}{L} \ln \frac{1}{R}+\alpha .
$$

Here $\Gamma$ is the optical confinement factor, $L$ is the laser cavity length, $R$ is the reflectivity of the mirrors, and $\alpha$ is the nonradiative absorption loss per unit length. By choosing $L$ to be large the mirror losses can be effectively eliminated. The photon losses $\alpha$ result mainly from free-carrier absorption. At low photon energies $(\sim 0.1 \mathrm{eV})$ these losses are prohibitively high in heavily doped material. At higher energies, though, the losses are greatly reduced. We take the free-carrier contribution to $\alpha$ to be $35 \mathrm{~cm}^{-1}$ at $0.5 \mathrm{eV}$, in agreement with experimental studies on heavily doped GaAs. ${ }^{10}$ We include residual losses of $15 \mathrm{~cm}^{-1}$, bringing the total photon losses to $50 \mathrm{~cm}^{-1}$.

Wc have used a separate optical confinement structure with a $2400 \AA \mathrm{In}_{0.53} \mathrm{Ga}_{0.47} \mathrm{As}$ cladding region bounded by InP. This yields a confinement factor of $\Gamma=0.00785$. Note that the confinement would be even lower for a longer wavelength device. Hence, it is essential to maximize the subband energy separation in order to maximize the optical confinement and minimize the free-carrier absorption.

With these parameters we obtain a value of $120 \mathrm{~cm}^{-1}$ for $\Gamma_{\beta}$, well in excess of the minimum modal gain of 50 $\mathrm{cm}^{-}$. However, it should be pointed out that this value is critically dependent on the linewidth of the intersubband transitions. In addition, the peak gain is directly proportional to the carrier density in the second subband at the second resonance. Our calculation, which includes selfconsistency and effective-mass changes in the well and barriers, gives a value of $1.9 \times 10^{11} \mathrm{~cm}^{-2}$ for this parameter. Again, a factor of 3 change in this value would prohibit the structure from lasing. Hence, we see that the system is extremely sensitivc to the carrier density and the intersubband relaxation time and both must be strictly controlled.

In order for the device to lase, the lower energy subband must remain unpopulated. This requires that the escape time $\tau_{1}$ for carriers to tunnel out of the first subband be less than the stimulated emission lifetime $\tau_{\text {stim }}$ $=\left[\left(\Gamma_{c} / n_{r}\right) g\right]^{-1}=1 \mathrm{ps}$. From the widths $\Delta E_{1}$ and $\Delta E_{2}$ of the transmission coefficients at the first and second resonances, we have calculated the escape times $\tau_{1,2}$ $=\hbar / q \Delta E_{1,2}$, and we obtain $\tau_{1}=0.34$ ps $<\tau_{\text {stim }}$ and $\tau_{2}=3.3$ fs $<\tau_{\text {stim }}$. Hence, the lower state will be depleted rapidly enough to maintain population inversion and the upper state will be populated by current injection much more rapidly than it will be depleted by stimulated emission. In addition, the spontaneous emission lifetime $\tau_{\mathrm{sp}}$ should far exceed the stimulated emission lifetime for the transition. Using the well-known spontaneous lifetime formula (centimeters-grams-seconds units) ${ }^{11}$

$$
\frac{1}{\tau_{\mathrm{sp}}}=\frac{4 n_{r}(\hbar \omega)^{3} e^{2}\left|M_{21}\right|^{2}}{3 \hbar^{4} c^{3}},
$$

we calculate $\tau_{\mathrm{sp}}=3.2 \mathrm{~ns} \gg \tau_{\mathrm{stim}}$. Thus, all the relevant lifetimes and rates are such that the device can operate as an 
infrared laser. Lastly, we remark that these calculations were done at $T=300 \mathrm{~K}$. If the temperature is decreased, the peak current and the associated carrier concentration at the second resonance is relatively unchanged. Hence, the temperature dependence of the device is expected to be small.

Resonant tunneling diodes may also be applied as light modulators. For an interband modulator, only the boundstate device is suitable since the absorption energy of the quantum well must be less than that of the surrounding waveguide region. Typical applications require a 5 to 1 change in light intensity, meaning that in the "off" mode the device must absorb $80 \%$ of the incident light. The optical absorption behavior is given by

$$
I(l)=I(0) e^{-\Gamma \alpha l},
$$

where $l$ is the distance through which the light has traversed the optically active region, $I(l)$ is the light intensity at the distance $l, I(0)$ is the initial light intensity, $\alpha$ is the optical absorption coefficient (typically $\sim 10^{4} \mathrm{~cm}^{-1}$ for any quantum well system), and $\Gamma$ is again the percentage of a given cross section of the light bcam that sees the optically active region. In conventional quantum-confined stark effect (QCSE) modulators the light is incident along the growth direction (normal to the layers), so almost all of a given cross section of the photon beam sees the quantum wells and we may take $\Gamma \sim 1$. In order to achieve a long absorption length $l$ with a single quantum well it is necessary to propagate the light along the quantum well in the waveguide geometry. This again requires a separate optical confinement structure. We have determined that a $700 \AA \mathrm{A}$ GaAs cladding region bounded by $\mathrm{Al}_{0.6} \mathrm{Ga}_{0.4}$ As provides an optimal confinement of $\Gamma=0.025$ at a transition energy of $1.3 \mathrm{eV}$. In order to obtain a 5:1 modulation ratio satisfying Eq. (5) for this device we require an absorption length $l \sim 40 \mu \mathrm{m}$.

We calculate the excitonic properties of the boundstate device under an arbitrary bias by using the nonvariational numerical technique described in Ref. 12. At zero bias, the well is filled with free carriers and the excitonic absorption is screened out. As a bias is applied, the well empties out and excitonic transitions can take place. These effects are manifested in the absorption profile for the bound-state device shown in Fig. 3. As can be seen from Figs. 2 and 3, the resulting carrier-concentration change of two orders of magnitude dramatically changes the absorption at the excitonic peak; the bound-state carrier concentration is calculated from the bound eigenstates in the quantum well. ${ }^{13}$ Note that we see two heavy-hole exciton peaks because the strain in the well has made the first two valence subbands of heavy-hole character. We calculate the absorption spectra from the Fermi golden rule in the dipole approximation. ${ }^{12}$ These calculations were done at a temperature of $300 \mathrm{~K}$. At lower temperatures the exciton becomes quenched at a much smaller carrier density

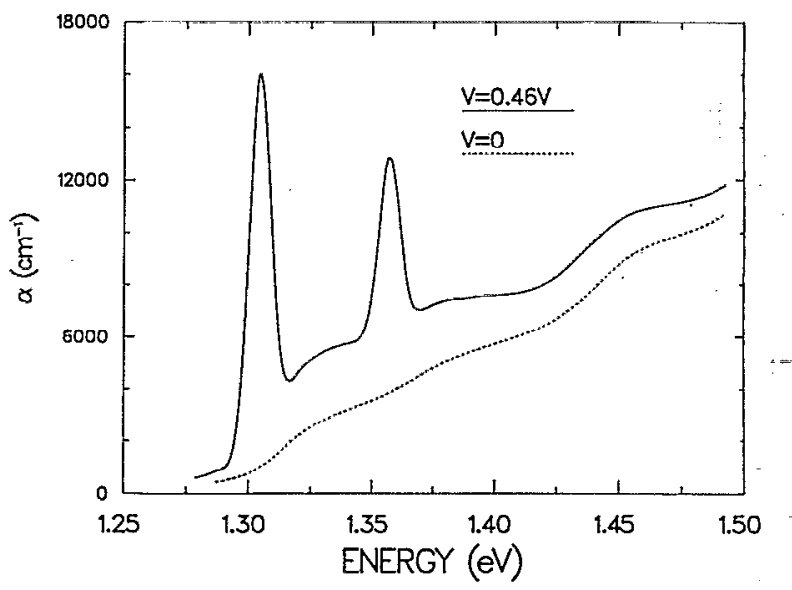

FIG. 3. Absorption spectra for $\hat{z}$-polarized light in the bound-state resonant tunneling diode at zero bias and $V=0.46 \mathrm{~V}$.

$\left(\sim 10^{10} / \mathrm{cm}^{2}\right.$ at $T=0$, for example). ${ }^{14}$ Hence, it will become more difficult to bias the device so that enough carriers tunnel out of the well to allow absorption, and the performance of the modulator will be degraded.

In conclusion, we have investigated the possible optical applications of resonant tunneling diodes. We find that a conventional RTD structure can function as a laser if the intersubband linewidth is small enough $(\sim 5 \mathrm{meV})$ and the carrier concentration at the second resonance is large enough $\left(\sim 2 \times 10^{11} \mathrm{~cm}^{-2}\right)$. In addition, the transition energy must be maximized $(\sim 0.5 \mathrm{eV})$ to obtain a high optical confinement factor and low free-carrier losses. Interband excitonic transilions may also be exploited in the bound-state device to produce an optical modulator in the waveguide geometry. We find that a typical modulation ratio of 5:1 may be obtained with a $40 \mu \mathrm{m}$ device.

The authors would like to thank Professor Glen A. B. Feak for the use of his program to calculate confinement factors. This work was supported by the Army URI. program (Grant No. DAAL03-87-K-0007) and the ONR under Contract No. N00014-90-J-1831.

${ }^{1}$ R. K. Mains and G. I. Haddad, J. Appl. Phys. 64, 3564 (1988).

${ }^{2}$ R. K. Mains, J. P. Sun, and G. I. Haddad, Appl. Phys. Lett. 55, 371 (1989).

${ }^{3}$ R. K. Mains, I. Mehdi, and G. I. Haddad, Appl. Phys. Lett. 55, 2631 (1989).

${ }^{4}$ P. F. Yuh and K. L. Wang, Appl. Phys. Lett. 51, 1404 (1987).

${ }^{5}$ H. C. Liu, J. Appl. Phys. 63, 2856 (1988).

${ }^{6}$ I. Mehdi, G. I. Haddad, and R. Mains, Superlatt. Microstruct. 5, 443 (1989).

${ }^{7}$ S. Borenstain and J. Katz, Appl. Phys. Lett. 55, 654 (1989).

${ }^{8}$ D. Ahn and S. L. Chuang, Phys. Rev. B 35, 4149 (1987).

${ }^{y}$ L. C. West and S. J. Eglash, Appl. Phys. Lett. 46, 1156 (1985).

${ }^{10}$ W. G. Spitzer and J. M. Whelan, Phys. Rev. 114, 59 (1959).

${ }^{11}$ See, for example, the discussion in J. J. Sakurai, Advanced Quantum Mechanics (Addison-Wesley, New York, 1967).

${ }^{12}$ J. P. Loehr and J. Singh, Phys. Rev. B 42, 7154 (1990).

${ }^{13}$ G. I. Haddad, U. K. Reddy, J. P. Sun, and R. K. Mains, Superlatt. Microstruct. 7, 369 (1990).

${ }^{14}$ G. D. Sanders and Y. C. Chang, Phys. Rev. B 35, 1300 (1987). 